\title{
CONTROLE DE SISTEMAS LINEARES COM COMUTAÇÃO
}

\author{
Grace S. Deaecto* \\ grace@dsce.fee.unicamp.br
}

\author{
José C. Geromel* \\ geromel@dsce. fee.unicamp.br
}

* DSCE / Faculdade de Engenharia Elétrica e de Computação

UNICAMP, CP 6101, 13083 - 970

Campinas, SP, Brasil

\begin{abstract}
This work aims at studying the stability of switched linear systems with impulsive disturbances and at designing a state feedback switched control. The conditions are based on Lyapunov-Metzler inequalities, which assure that stability is preserved even if the system evolves on a sliding mode. A slight modification of these inequalities is introduced to cope with a guaranteed cost performance. In order to analyze the quality of the proposed solution, a lower bound for the actual cost is calculated. The theoretical results are applied to a multiobjective $\mathcal{H}_{2}$ control design problem with a number of possibly conflicting criteria. It is shown that the use of switched linear systems theory in multiobjective $\mathcal{H}_{2}$ control problems provides better results than others methods available in the literature. Academic examples are used for illustration.
\end{abstract}

KEYWORDS: switched systems, $\mathcal{H}_{2}$ control, continuoustime systems, Lyapunov-Metzler inequalities.

\section{RESUMO}

Este trabalho tem como objetivo o estudo de estabilidade de sistemas lineares com comutação sujeitos à perturbações impulsivas tendo em vista o projeto de controle via realimentação de estado com comutação. As condições obtidas são baseadas nas desigualdades de Lyapunov-Metzler, que asse-

\footnotetext{
Artigo submetido em 06/03/2007

1a. Revisão em 09/09/2008

2a. Revisão em 29/09/2008

Aceito sob recomendação do Editor Associado

Prof. José Roberto Castilho Piqueira
}

guram a estabilidade mesmo quando o sistema evoluir sobre um modo deslizante. Estas desigualdades permitem levar em conta um custo garantido de desempenho. De forma a analisar a qualidade do resultado, um limitante inferior para o custo verdadeiro é determinado. A teoria desenvolvida é aplicada em um problema de controle $\mathcal{H}_{2}$ multiobjetivo com critérios possivelmente conflitantes. É mostrado que, neste contexto, o uso de controle com comutação fornece resultados melhores quando comparados com outros métodos encontrados na literatura. Exemplos acadêmicos são usados para ilustração.

PALAVRAS-CHAVE: sistemas com comutação, controle $\mathcal{H}_{2}$, sistemas a tempo contínuo, desigualdades de LyapunovMetzler.

\section{INTRODUÇÃO}

Nos últimos anos, o interesse no estudo de sistemas com comutação tem sido bastante significativo. A motivação devese ao fato de que a comutação entre subsistemas pode melhorar o desempenho global, além de permitir o surgimento de propriedades importantes que não são encontradas nos subsistemas isolados. Em princípio, a teoria de sistemas lineares com comutação pode ser dividida em dois grupos. No primeiro a regra de comutação, denotada $\sigma(\cdot)$, é independente dos estados e corresponde a uma incerteza variante no tempo. Neste caso, as condições de estabilidade devem garantir a robustez para qualquer $\sigma(\cdot) \in\{1,2, \cdots, N\}$, sendo $N$ a quantidade de subsistemas. Veja (Hespanha e Morse, 2002) para a síntese de controladores que estabilizam sistemas com comutação em um contexto bastante geral. O segundo grupo é caracterizado pelo fato de $\sigma(\cdot)$ ser uma variável de controle que 
pode, inclusive, depender do estado do sistema em estudo. O objetivo consiste na determinação de uma regra de comutação $\sigma(\cdot)$ de tal forma a assegurar a estabilidade assintótica global. Em (Liberzon e Morse, 1999), (Liberzon, 2003), (DeCarlo et al., 2000), (Wirth, 2005), (Shorten et al., 2007) o leitor pode encontrar conjuntos de resultados bastante completos sobre sistemas lineares com comutação a tempo contínuo, em especial, para o caso de comutação envolvendo apenas dois subsistemas lineares. O artigo (Hespanha, 2005) utiliza extensões do Princípio da Invariância de LaSalle e fornece uma discussão interessante sobre resultados referentes à estabilidade uniforme de sistemas com comutação. Sistemas com comutação a tempo discreto são tratados em (Geromel e Colaneri, 2006b) e resultados sobre a construção de funções de Lyapunov estão disponíveis em (Daafouz e Bernussou, 2001), (Johansson e Rantzer, 1998).

Este trabalho tem como objetivo principal generalizar as condições de estabilidade obtidas em (Geromel e Colaneri, 2006a) para tratar sistemas com comutação mais gerais, sujeitos à perturbações impulsivas e realizar o projeto conjunto de uma regra de comutação $\sigma(t)=g(x(t)) \in\{1,2, \cdots, N\}$ e de ganhos de realimentação de estado $\left\{K_{1}, \cdots, K_{N}\right\}$ que permitem construir a lei de controle $u(t)=K_{\sigma(t)} x(t)$. Mostramos que as condições de estabilidade dependem da solução de um conjunto de desigualdades de Lyapunov-Metzler mais gerais do que aquelas introduzidas em (Geromel e Colaneri, 2006a). Estas desigualdades possuem natureza nãoconvexa devido à existência de produtos de variáveis, entre matrizes simétricas definidas positivas $\left\{P_{1}, \cdots, P_{N}\right\}$ e uma matriz de Metzler П. Assim sendo, não é possível resolvêlas através da utilização de ferramentas disponíveis na literatura para tratar LMIs, (Boyd et al., 1994). No entanto, apresentamos uma versão alternativa mais simples, embora mais conservadora, que é baseada nos resultados de (Geromel e Colaneri, 2006a) e consiste na utilização de uma subclasse de matrizes de Metzler caracterizada por apresentar elementos iguais na diagonal principal. Neste caso, a solução das referidas desigualdades não-convexas é conseguida através da manipulação de LMIs em conjunto com uma busca unidimensional. Em seguida, o problema de minimização de um custo garantido é tratado e, para evidenciar a qualidade do resultado final, calculamos limitantes superior e inferior (ver (Spinelli et al., 2006) para uma proposta alternativa) do custo ótimo.

As condições obtidas garantem a estabilidade inclusive na presença de possíveis modos deslizantes e não exigem que isoladamente cada subsistema seja estável. A teoria desenvolvida é ilustrada através de alguns exemplos. Em um deles, projetamos o controle para dois subsistemas instáveis e não controláveis para mostrar que a atuação conjunta de ambas as variáveis de controle, a saber, a regra de comutação e os ganhos de realimentação de estado, faz com que a origem do sistema em malha fechada seja globalmente assintoticamente estável. Em outro exemplo, projetamos um controle $\mathcal{H}_{2}$ multiobjetivo com comutação e o comparamos com as alternativas de controle propostas em (Khargonekar e Rotea, 1991) e (Eker e Malmborg, 1999). Resultados complementares, bem como uma discussão mais detalhada daqueles aqui apresentados podem ser encontrados em (Deaecto, 2007).

A notação usada é padrão. Para matrizes ou vetores reais $\left(^{\prime}\right)$ indica o seu transposto. Para matrizes quadradas $\operatorname{tr}(X)$ denota a função traço de $X$. Para facilitar a notação de matrizes simétricas particionadas, o símbolo $(\bullet)$ denota genericamente cada bloco simétrico. O símbolo $\delta(t)$ denota o impulso unitário. A norma $l_{2}$ de uma trajetória $\xi(t)$ definida para todo $t \geq 0$ é dada por $\|\xi\|_{2}^{2}:=\int_{0}^{\infty} \xi(t)^{\prime} \xi(t) d t$. O conjunto $\{1, \cdots, N\}$ é denotado por $\mathbb{K}$. Finalmente, a convolução de dois sinais $\xi_{1}(t)$ e $\xi_{2}(t)$ definidos para todo $t \geq 0$ é indicada por $\xi_{1} * \xi_{2}$.

\section{ESTABILIDADE E DESEMPENHO}

Para dar embasamento à teoria a ser desenvolvida em seguida, alguns resultados preliminares já apresentados em (Geromel e Colaneri, 2006a) são expostos. Considere o sistema

$$
\dot{x}(t)=A_{\sigma(t)} x(t), x(0)=x_{0}
$$

definido para todo $t \geq 0$, em que $x(t) \in \mathbb{R}^{n}$ é o estado e é suposto disponível para a realimentação, $\sigma(t)$ é a regra de comutação e $x_{0} \in \mathbb{R}^{n}$ é a condição inicial. Em cada instante de tempo a regra de comutação $\sigma(t) \in \mathbb{K}$ seleciona uma matriz $A_{\sigma(t)} \in \mathbb{R}^{n \times n}$ dentre aquelas pertencentes ao conjunto $\left\{A_{1}, \ldots, A_{N}\right\}$. As condições de estabilidade global, isto é, as condições sob as quais a origem $x=0$ é um ponto de equilíbrio globalmente assintoticamente estável, são obtidas adotando-se a seguinte função de Lyapunov

$$
v(x)=\min _{i \in \mathbb{K}} x^{\prime} P_{i} x=\min _{\lambda \in \Lambda}\left(\sum_{i=1}^{N} \lambda_{i} x^{\prime} P_{i} x\right)
$$

sendo $P_{i}, \forall i \in \mathbb{K}$ matrizes simétricas definidas positivas e $\lambda \in \mathbb{R}^{N}$ um elemento do conjunto convexo $\Lambda$,

$$
\Lambda=\left\{\lambda \in \mathbb{R}^{N}: \sum_{i=1}^{N} \lambda_{i}=1, \lambda_{i} \geq 0\right\}
$$

Um aspecto relevante a ser considerado é que a função (2) não é diferenciável para todo $x \in \mathbb{R}^{n}$. De fato, pode-se verificar $^{1}$ que ela deixa de ser diferenciável em todos os pontos onde a minimização indicada em (2) não for única. Para facilitar os desenvolvimentos posteriores, definimos o conjunto de índices $I(x)=\left\{i \in \mathbb{K}: v(x)=x^{\prime} P_{i} x\right\} \subset \mathbb{K}$ e notamos

\footnotetext{
${ }^{1}$ Para maiores detalhes o leitor deve consultar o apêndice.
} 
que para os casos em que a minimização (2) não for única, o conjunto $I(x)$ contém mais de um elemento.

Nosso objetivo é encontrar uma regra de comutação $g(x)$ : $\mathbb{R}^{n} \rightarrow \mathbb{K}$, e as condições para que $\sigma(t)=g(x(t))$ faça com que a origem do sistema (1) seja um ponto de equilíbrio globalmente assintoticamente estável. O teorema dado a seguir apresenta estas condições e, além disso, estabelece um limitante superior para um custo que depende quadraticamente das variáveis de estado do sistema em malha fechada. Sua prova decorre da utilização da função de Lyapunov (2) e de uma classe de matrizes de Metzler denotada por $\mathcal{M}$ e composta por todas as matrizes $\Pi \in \mathbb{R}^{N \times N}$ tais que

$$
\left\{\begin{array}{ccrl}
\pi_{j i} \geq 0 & , & i \neq j \in \mathbb{K} \times \mathbb{K} \\
\sum_{j=1}^{N} \pi_{j i}=0 & , & i \in \mathbb{K}
\end{array}\right.
$$

Observe, como uma conseqüência imediata, que para qualquer matriz desta classe, todos os elementos da sua diagonal principal são não-positivos, isto é $\pi_{i i} \leq 0$ para todo $i \in \mathbb{K}$.

Teorema 1 Considere $Q_{i}$ para todo $i \in \mathbb{K}$ matrizes simétricas semi-definidas positivas. Se existirem um conjunto de matrizes simétricas definidas positivas $\left\{P_{1}, \ldots, P_{N}\right\}$ e uma matriz $\Pi \in \mathcal{M}$ satisfazendo as seguintes desigualdades de Lyapunov-Metzler

$$
A_{i}^{\prime} P_{i}+P_{i} A_{i}+\sum_{j=1}^{N} \pi_{j i} P_{j}+Q_{i}<0, i \in \mathbb{K}
$$

então a regra de comutação

$$
\sigma(t)=\arg \min _{i \in \mathbb{K}} x(t)^{\prime} P_{i} x(t)
$$

faz com que a origem do sistema (1) seja um ponto de equilíbrio globalmente assintoticamente estável. Ademais, para qualquer condição inicial a seguinte desigualdade

$$
\int_{0}^{\infty} x(t)^{\prime} Q_{\sigma(t)} x(t) d t<\min _{i \in \mathbb{K}} x_{0}^{\prime} P_{i} x_{0}
$$

é verdadeira.

Prova: Embora a prova já tenha sido apresentada em (Geromel e Colaneri, 2006a), vamos aqui repeti-la para comodidade do leitor. Devido ao fato de que a função (2) não é diferenciável para todo $x \in \mathbb{R}^{n}$, calculamos sua derivada de Dini, dada por (Garg, 1998)

$$
D^{+} v(x(t))=\lim _{h \rightarrow 0^{+}} \sup \frac{v(x(t+h))-v(x(t))}{h}
$$

Assim sendo, considerando que em um instante de tempo genérico temos $\sigma(t)=i \in I(x(t))$, os desenvolvimentos apresentados no apêndice levam a

$$
\begin{aligned}
D^{+} v(x(t)) & =\min _{l \in I(x(t))} x(t)^{\prime}\left(A_{i}^{\prime} P_{l}+P_{l} A_{i}\right) x(t) \\
& \leq x(t)^{\prime}\left(A_{i}^{\prime} P_{i}+P_{i} A_{i}\right) x(t)
\end{aligned}
$$

Lembrando que $x(t)^{\prime} P_{j} x(t) \geq x(t)^{\prime} P_{i} x(t)$ e que $\pi_{j i} \geq 0$ para todo $j \neq i \in \mathbb{K}$, utilizando (5) obtemos

$$
\begin{aligned}
D^{+} v(x(t)) & <x(t)^{\prime}\left(-\sum_{j=1}^{N} \pi_{j i} P_{j}-Q_{i}\right) x(t) \\
& <-\left(\sum_{j=1}^{N} \pi_{j i}\right) x(t)^{\prime} P_{i} x(t)-x(t)^{\prime} Q_{i} x(t) \\
& <-x(t)^{\prime} Q_{i} x(t) \\
& <0
\end{aligned}
$$

fazendo com que a origem do sistema (1) seja globalmente assintoticamente estável. A determinação do limitante superior segue também da desigualdade (10) pois, lembrando que $\sigma(t)=i$, temos

$$
D^{+} v(x(t))<-x(t)^{\prime} Q_{\sigma(t)} x(t)
$$

Integrando ambos os lados desta desigualdade entre os instantes 0 e $t$, fazendo em seguida $t \rightarrow \infty$ obtemos (7), já que a estabilidade assintótica global do sistema (1) implica $v(x(\infty))=0$.

Um ponto relevante a ser levado em consideração é que as condições definidas em (Geromel e Colaneri, 2006a) asseguram a estabilidade do sistema inclusive na presença de possíveis modos deslizantes, já que para cada $t \geq 0, I(x(t))$ pode conter mais de um elemento. Neste caso, $\sigma(t)$ utiliza apenas um deles selecionado arbitrariamente dentre os elementos de $I(x(t))$. Outro ponto a ser levado em consideração é que as condições apresentadas no Teorema 1 não exigem nenhuma propriedade de estabilidade das matrizes $\left\{A_{1}, \cdots, A_{N}\right\}$ tomadas individualmente. De fato, como $\pi_{j i} \geq 0$ para $i \neq j$, uma condição necessária para que as desigualdades (5) sejam factíveis é que

$$
\left(A_{i}+\frac{\pi_{i i}}{2} I\right)^{\prime} P_{i}+P_{i}\left(A_{i}+\frac{\pi_{i i}}{2} I\right)<0
$$

para todo $i \in \mathbb{K}$. Como $P_{i}>0$ para todo $i \in \mathbb{K}$, isto só pode ocorrer se as matrizes $A_{i}+\left(\pi_{i i} / 2\right) I, i \in \mathbb{K}$ forem assintoticamente estáveis. Além disso, tendo em vista que $\pi_{i i} \leq 0, i \in \mathbb{K}$, a factibilidade de (12) não exige a estabilidade de nenhuma das matrizes do conjunto $\left\{A_{1}, \cdots, A_{N}\right\}$. Em outras palavras, as desigualdades de Lyapunov-Metzler (5) podem ser satisfeitas mesmo que as matrizes $A_{i}, i \in \mathbb{K}$ não sejam assintoticamente estáveis. Este importante aspecto será ilustrado através de exemplos.

Vale ressaltar que a escolha da função de Lyapunov não é única. As condições para a estabilidade do sistema (1) podem, com uma certa condição adicional, ser obtidas através da utilização de uma função de Lyapunov diferente. Uma de- 
las, ver (Liberzon, 2003) para maiores detalhes, é a seguinte

$$
V(x)=\max _{i \in \mathbb{K}} x^{\prime} P_{i} x=\max _{\lambda \in \Lambda}\left(\sum_{i=1}^{N} \lambda_{i} x^{\prime} P_{i} x\right)
$$

em que $P_{i}$ é uma matriz simétrica definida positiva pertencente ao conjunto $\left\{P_{1}, \ldots, P_{N}\right\}$. De maneira similar ao caso anterior definimos $\mathcal{I}(x)=\left\{i \in \mathbb{K}: V(x)=x^{\prime} P_{i} x\right\} \mathrm{e}$ consideramos o conjunto de desigualdades matriciais

$$
A_{i}^{\prime} P_{i}+P_{i} A_{i}-\sum_{j=1}^{N} \pi_{j i} P_{j}+Q_{i}<0, i \in \mathbb{K}
$$

que ao serem resolvidas em relação às matrizes simétricas definidas positivas $\left\{P_{1}, \cdots, P_{N}\right\}$ e à matriz de Metzler $\Pi$, permitem definir a regra de comutação

$$
\sigma(t)=\arg \max _{i \in \mathbb{K}} x(t)^{\prime} P_{i} x(t)
$$

Seria de se esperar, dada a simetria min / max das soluções propostas, que ambas fornecessem sistemas globalmente assintoticamente estáveis. Infelizmente, este não é o caso, a não ser que assumamos que o conjunto $\mathcal{I}(x(t))$ contenha apenas um elemento para todo $t \geq 0$. Para verificar esta afirmação, considerando que em um instante genérico $t \geq 0$ temos $\sigma(t)=i \in \mathcal{I}(x(t))$, a partir dos resultados apresentados no apêndice obtemos

$$
\begin{aligned}
D^{+} V(x(t)) & =\max _{l \in \mathcal{I}(x(t))} x(t)^{\prime}\left(A_{i}^{\prime} P_{l}+P_{l} A_{i}\right) x(t) \\
& =x(t)^{\prime}\left(A_{i}^{\prime} P_{i}+P_{i} A_{i}\right) x(t)
\end{aligned}
$$

Novamente, lembrando que $x(t)^{\prime} P_{j} x(t) \leq x(t)^{\prime} P_{i} x(t)$ e que $\pi_{j i} \geq 0$ para todo $j \neq i \in \mathbb{K}$, utilizando (14), vem

$$
\begin{aligned}
D^{+} V(x(t)) & <x(t)^{\prime}\left(\sum_{j=1}^{N} \pi_{j i} P_{j}-Q_{i}\right) x(t) \\
& <\left(\sum_{j=1}^{N} \pi_{j i}\right) x(t)^{\prime} P_{i} x(t)-x(t)^{\prime} Q_{i} x(t) \\
& <-x(t)^{\prime} Q_{i} x(t) \\
& <0
\end{aligned}
$$

o que comprova a estabilidade assintótica global do sistema com a regra de comutação (15). Note que a segunda igualdade em (16) depende fortemente do fato de existir apenas um elemento em $\mathcal{I}(x(t))$ em todo $t \geq 0$. Se esta hipótese não se verificar, por exemplo, na eventual ocorrência de modos deslizantes, $\mathcal{I}(x(t))$ necessariamente contém mais de um elemento e a estabilidade do sistema não pode ser estabelecida. Nesta situação, verifica-se através de exemplos que o sistema torna-se instável, (Deaecto, 2007). Entretanto, em alguns casos, a estabilidade assintótica ainda pode ser verificada a posteriori através de testes suplementares que permitam assegurar que o conjunto $\mathcal{I}(x(t))$ contém apenas um elemento, (Ishii et al., 2004). Infelizmente, estes testes são muito difíceis de serem aplicados sobretudo quando o número de subsistemas é maior do que dois.

De acordo com o Teorema 1, as condições para a estabilidade assintótica de (1) dependem da solução das desigualdades de Lyapunov-Metzler (5). Estas desigualdades possuem natureza não-convexa devido ao produto das variáveis $\left(\Pi,\left\{P_{1}, \ldots, P_{N}\right\}\right)$ e não é possível resolvê-las através de rotinas numéricas disponíveis na literatura para a solução de LMIs. No entanto, em (Geromel e Colaneri, 2006a) foi proposta uma versão mais conservadora que pode ser resolvida utilizando-se LMIs e uma busca unidimensional. Ela é obtida quando restringimos as matrizes de Metzler do Teorema 1 a terem uma estrutura particular com todos os elementos iguais na diagonal principal.

Corolário 2 Considere $Q_{i}$ para todo $i \in \mathbb{K}$ matrizes simétricas semi-definidas positivas. Se existirem um conjunto de matrizes simétricas definidas positivas $\left\{P_{1}, \ldots, P_{N}\right\}$ e um escalar positivo $\gamma$ satisfazendo as desigualdades modificadas de Lyapunov-Metzler

$$
A_{i}^{\prime} P_{i}+P_{i} A_{i}+\gamma\left(P_{j}-P_{i}\right)+Q_{i}<0, j \neq i \in \mathbb{K} \times \mathbb{K}
$$

então a regra de comutação (6) faz com que a origem do sistema (1) seja um ponto de equilíbrio globalmente assintoticamente estável e a desigualdade (7) seja satisfeita.

Prova: Considerando a subclasse de matrizes de Metzler com elementos iguais na diagonal principal, isto é $\pi_{i i}=-\gamma$, para todo $i \in \mathbb{K}$ temos

$$
\gamma^{-1} \sum_{j \neq i=1}^{N} \pi_{j i}=1
$$

Levando em conta que $\pi_{j i} \geq 0$ para todo $i \neq j \in \mathbb{K} \times \mathbb{K}$, multiplicando (18) por $\pi_{j i}$, somando para todos os elementos $i \neq j$ e finalmente multiplicando por $\gamma^{-1}$, obtemos

$$
\begin{aligned}
A_{i}^{\prime} P_{i}+P_{i} A_{i}+Q_{i} & <-\sum_{j \neq i=1}^{N} \pi_{j i}\left(P_{j}-P_{i}\right) \\
& <-\sum_{j=1}^{N} \pi_{j i} P_{j}
\end{aligned}
$$

para todo $i \in \mathbb{K}$. Ou seja, as desigualdades de LyapunovMetzler (5) são verificadas e a prova decorre imediatamente do Teorema 1.

Embora mais conservadoras do que as desigualdades de Lyapunov-Metzler, estas introduzidas no Corolário 2 são 
muito mais simples de serem resolvidas pois se reduzem a $N$ LMIs acopladas para cada valor de $\gamma>0$ fixo. Ademais, a condição necessária para a existência de solução continua a não exigir que as matrizes do conjunto $\left\{A_{1}, \cdots, A_{N}\right\}$ sejam assintoticamente estáveis.

\section{PERTURBAÇÕES IMPULSIVAS}

Considere um sistema com comutação e condições iniciais nulas, cuja realização no espaço de estado é dada por

$$
\begin{aligned}
\dot{x}(t) & =A_{\sigma(t)} x(t)+G_{\sigma(t)} w(t) \\
\xi(t) & =C_{\sigma(t)} x(t)
\end{aligned}
$$

em que $x(t) \in \mathbb{R}^{n}$ é o estado, $w(t) \in \mathbb{R}^{m}$ é a perturbação externa e $\xi(t) \in \mathbb{R}^{p}$ é a saída controlada. A regra de comutação $\sigma(t)=i \in \mathbb{K}$ seleciona um determinado subsistema definido por $\left(A_{i}, G_{i}, C_{i}\right)$. Para cada $\theta=1, \cdots, m$, uma entrada impulsiva da forma $w(t)=e_{\theta} \delta(t)$, com $e_{\theta}$ representando a $\theta$-ésima coluna da matriz identidade $m \times m$, é aplicada e a correspondente saída controlada $\xi^{\theta}(t)$ é obtida, o que permite determinar $\left\|\xi^{\theta}\right\|_{2}^{2}$. Desta forma, definimos o custo total associado a uma determinada regra de comutação $\sigma(t)$ como sendo a soma das contribuições dos custos para $\theta=1, \cdots, m$, ou seja

$$
\Theta(\sigma)=\sum_{\theta=1}^{m}\left\|\xi^{\theta}\right\|_{2}^{2}
$$

É importante salientar que na ausência de comutação, isto é, quando $\sigma(t)=i \in \mathbb{K}$ para todo $t \geq 0$, a quantidade $\Theta(\sigma)$ coincide com o quadrado da norma $\mathcal{H}_{2}$ da função de transferência entre a entrada $w$ e a saída $\xi$ do sistema dinâmico linear com representação de estado definida pelas matrizes $\left(A_{i}, G_{i}, C_{i}\right)$, (Colaneri et al., 1997). O teorema a seguir apresenta condições para a estabilidade assintótica global do sistema (21)-(22), bem como um limitante superior do custo funcional (23).

Teorema 3 Se existirem um conjunto de matrizes simétricas definidas positivas $\left\{P_{1}, \ldots, P_{N}\right\}$ e uma matriz $\Pi \in \mathcal{M}$ satisfazendo as seguintes desigualdades de Lyapunov-Metzler

$$
A_{i}^{\prime} P_{i}+P_{i} A_{i}+\sum_{j=1}^{N} \pi_{j i} P_{j}+C_{i}^{\prime} C_{i}<0, i \in \mathbb{K}
$$

então a regra de comutação (6) faz com que o ponto de equilíbrio $x=0$ do sistema (21)-(22) seja globalmente assintoticamente estável. Ademais, a seguinte desigualdade

$$
\Theta(\sigma)<\min _{i \in \mathbb{K}} \operatorname{tr}\left(G_{\sigma(0)}^{\prime} P_{i} G_{\sigma(0)}\right)
$$

é verdadeira.
Prova: Podemos observar que para a mesma regra de comutação, a solução de (21) com $x(0)=0$ e $w(t)=e_{\theta} \delta(t)$ é igual à solução de $(1) \operatorname{com} x(0)=G_{\sigma(0)} e_{\theta}$, pois ambas satisfazem

$$
x(t)=\int_{0}^{t} A_{\sigma(\tau)} x(\tau) d \tau+G_{\sigma(0)} e_{\theta}
$$

Esta propriedade permite utilizar diretamente os resultados do Teorema 1 no sistema (21)-(22). Substituindo as matrizes $Q_{i}$ pelas matrizes $C_{i}^{\prime} C_{i}$ para todo $i \in \mathbb{K}$ obtemos as desigualdades (24) que asseguram a estabilidade assintótica bem como a validade do limitante superior

$$
\left\|\xi^{\theta}\right\|_{2}^{2}<\min _{i \in \mathbb{K}} e_{\theta}^{\prime} G_{\sigma(0)}^{\prime} P_{i} G_{\sigma(0)} e_{\theta}
$$

Conseqüentemente, somando as parcelas para $\theta=1, \cdots, m$ determinamos

$$
\begin{aligned}
\Theta(\sigma) & <\sum_{\theta=1}^{m} \min _{i \in \mathbb{K}} e_{\theta}^{\prime} G_{\sigma(0)}^{\prime} P_{i} G_{\sigma(0)} e_{\theta} \\
& <\min _{i \in \mathbb{K}} \sum_{\theta=1}^{m} e_{\theta}^{\prime} G_{\sigma(0)}^{\prime} P_{i} G_{\sigma(0)} e_{\theta} \\
& <\min _{i \in \mathbb{K}} \operatorname{tr}\left(G_{\sigma(0)}^{\prime} P_{i} G_{\sigma(0)}\right)
\end{aligned}
$$

o que prova o teorema proposto.

Uma discussão relevante a respeito do resultado deste teorema refere-se à determinação do valor de $\sigma(0)$ a ser usado em (21)-(22). Note que no instante $t=0$, imediatamente anterior à aplicação do impulso, a regra de comutação

$$
\sigma(0)=\arg \min _{i \in \mathbb{K}} x(0)^{\prime} P_{i} x(0)
$$

não especifica $\sigma(0)$ pois $x(0)=0$. Ou seja, podemos escolher $\sigma(0)=\ell \in \mathbb{K}$ da forma mais conveniente para reduzir o custo garantido que aparece no lado direito de (25). Assim procedendo, obtemos

$$
\Theta(\sigma)<\min _{i, \ell \in \mathbb{K} \times \mathbb{K}} \inf _{\left(\Pi \in \mathcal{M}, P_{i}>0\right) \in \Phi} \operatorname{tr}\left(G_{\ell}^{\prime} P_{i} G_{\ell}\right)
$$

em que $\Phi$ representa o conjunto de todas as soluções factíveis de (24). O esforço computacional para resolver o problema de otimização indicado no lado direito de (30) pode ser reduzido impondo-se $\ell=i \in \mathbb{K}$. É claro que, assim procedendo, o resultado final é um limitante superior mais conservador. $\mathrm{O}$ corolário a seguir apresenta as condições para a estabilidade assintótica global do sistema (21)-(22) utilizando a subclasse de matrizes de Metzler com elementos iguais na diagonal principal.

Corolário 4 Se existirem um conjunto de matrizes simétricas definidas positivas $\left\{P_{1}, \ldots, P_{N}\right\}$ e um escalar positivo 
$\gamma$ satisfazendo as desigualdades modificadas de LyapunovMetzler

$$
A_{i}^{\prime} P_{i}+P_{i} A_{i}+\gamma\left(P_{j}-P_{i}\right)+C_{i}^{\prime} C_{i}<0, j \neq i \in \mathbb{K} \times \mathbb{K}
$$

então a regra de comutação (6) faz com que a origem do sistema (21)-(22) seja um ponto de equilíbrio globalmente assintoticamente estável e a desigualdade (25) seja satisfeita.

Prova: A demonstração segue, em linhas gerais, aquela do Corolário 2 e será, portanto, omitida.

Uma maneira de verificar se o custo garantido proposto no Teorema 3 é preciso consiste em determinar a folga eventualmente existente entre ambos os lados da desigualdade (25). Entretanto, como a determinação analítica do custo $\Theta(\sigma)$ é virtualmente impossível, propomos um limitante inferior para $\Theta(\sigma)$ de forma a viabilizar aquela verificação, ainda que não exatamente.

Teorema 5 Se existirem um conjunto de matrizes simétricas definidas positivas $\left\{P_{1}, \cdots, P_{N}\right\}$ e uma matriz $\Pi \in \mathcal{M}$ satisfazendo as seguintes desigualdades de Lyapunov-Metzler

$$
A_{q}^{\prime} P_{i}+P_{i} A_{q}+\sum_{j=1}^{N} \pi_{j i} P_{j}+C_{q}^{\prime} C_{q}>0, i, q \in \mathbb{K} \times \mathbb{K}
$$

então para qualquer regra de comutação que estabiliza o sistema (21)-(22), a seguinte desigualdade é verdadeira

$$
\Theta(\sigma)>\max _{i \in \mathbb{K}} \operatorname{tr}\left(G_{\sigma(0)}^{\prime} P_{i} G_{\sigma(0)}\right)
$$

Prova: Considerando que em um instante de tempo $t \geq 0$ temos $\sigma(t)=q \in \mathbb{K}$ sendo $\sigma(\cdot)$ uma regra de comutação qualquer que estabiliza assintoticamente o sistema (1), a derivada direcional da função de Lyapunov (13) permite concluir que

$$
\begin{aligned}
D^{+} V(x(t)) & =\max _{l \in \mathcal{I}(x(t))} x(t)^{\prime}\left(A_{q}^{\prime} P_{l}+P_{l} A_{q}\right) x(t) \\
& \geq x(t)^{\prime}\left(A_{q}^{\prime} P_{i}+P_{i} A_{q}\right) x(t)
\end{aligned}
$$

para todo $i \in \mathcal{I}(x(t))$. Por outro lado, levando em conta que $x(t)^{\prime} P_{i} x(t) \geq x(t)^{\prime} P_{j} x(t)$ e que $\pi_{j i} \geq 0$ para todo $j \neq i \in \mathbb{K}$, usando (32), obtemos

$$
\begin{aligned}
D^{+} V(x(t)) & >x(t)^{\prime}\left(-\sum_{j=1}^{N} \pi_{j i} P_{j}-C_{q}^{\prime} C_{q}\right) x(t) \\
& >-x(t)^{\prime} C_{q}^{\prime} C_{q} x(t) \\
& >-\xi(t)^{\prime} \xi(t)
\end{aligned}
$$

que após integração em ambos os lados fornece $V(x(0))-$ $V(x(\infty))<\|\xi\|_{2}^{2}$. Desta forma, com a condição inicial $x_{0}=$
$G_{\sigma(0)} e_{\theta} \in \mathbb{R}^{n}$ e levando em conta que a regra de comutação estabilizante faz com que $V(x(\infty))=0$, temos $\left\|\xi^{\theta}\right\|_{2}^{2}>$ $V\left(x_{0}\right)=\max _{i \in \mathbb{N}} x_{0}^{\prime} P_{i} x_{0}$. Conseqüentemente,

$$
\begin{aligned}
\Theta(\sigma) & >\sum_{\theta=1}^{m} \max _{i \in \mathbb{K}} e_{\theta}^{\prime} G_{\sigma(0)}^{\prime} P_{i} G_{\sigma(0)} e_{\theta} \\
& >\max _{i \in \mathbb{K}} \operatorname{tr}\left(G_{\sigma(0)}^{\prime} P_{i} G_{\sigma(0)}\right)
\end{aligned}
$$

o que prova o teorema proposto.

Note que é possível determinar o maior limitante inferior para $\Theta(\sigma)$ maximizando-se o lado direito de (33) o que nos leva à desigualdade

$$
\Theta(\sigma)>\max _{i \in \mathbb{K}} \sup _{\left(\Pi \in \mathcal{M}, P_{i}>0\right) \in \Omega} \operatorname{tr}\left(G_{\sigma(0)}^{\prime} P_{i} G_{\sigma(0)}\right)
$$

em que $\Omega$ representa o conjunto de todas as soluções factíveis de (32).

Como já foi comentado anteriormente, encontrar uma solução para as desigualdades (32) não é uma tarefa simples. A dificuldade reside no fato de que elas não são lineares devido à presença dos produtos de variáveis $\pi_{j i} P_{j}$ para todo $i, j \in \mathbb{K} \times \mathbb{K}$. Uma maneira de contornar esta dificuldade é considerar apenas matrizes $\Pi \in \mathcal{M}$ com elementos iguais na diagonal principal. O corolário seguinte apresenta formalmente este resultado.

Corolário 6 Se existirem um conjunto de matrizes simétricas definidas positivas $\left\{P_{1}, \cdots, P_{N}\right\}$ e um escalar positivo $\gamma$ satisfazendo as seguintes desigualdades modificadas de Lyapunov-Metzler

$$
A_{q}^{\prime} P_{i}+P_{i} A_{q}+\gamma\left(P_{j}-P_{i}\right)+C_{q}^{\prime} C_{q}>0,
$$

para todo $i \neq j \in \mathbb{K} \times \mathbb{K}$ e $q \in \mathbb{K}$, então para qualquer regra de comutação que estabiliza o sistema (21)-(22), a desigualdade (33) é verdadeira.

Prova: Trata-se de prova idêntica àquela do Corolário 2, sendo assim omitida.

O exemplo dado a seguir ilustra a teoria desenvolvida nesta seção. Nosso objetivo, em particular, é aplicar os resultados do Corolário 4 e do Corolário 6 para avaliar a folga existente entre os limitantes superior (25) e inferior (33) do custo $\Theta(\sigma)$ para uma regra de comutação específica.

Exemplo 1: Este exemplo foi proposto em (Geromel et al., 2008). Considere o sistema (21)-(22), constituído por $N=2$ subsistemas lineares definidos pelas matrizes

$$
A_{1}=\left[\begin{array}{rrr}
0 & 1 & 0 \\
0 & 0 & 1 \\
-2 & -2 & 0
\end{array}\right], A_{2}=\left[\begin{array}{rrr}
0 & 1 & 0 \\
0 & 0 & 1 \\
0 & -2 & -2
\end{array}\right]
$$




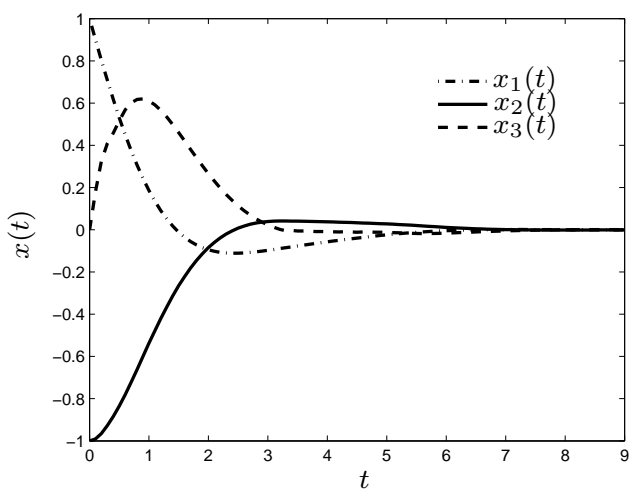

Figura 1: Estados do sistema - Exemplo 1

$$
\begin{gathered}
G=G_{1}=G_{2}=\left[\begin{array}{rr}
1 & 0 \\
-1 & -1 \\
0 & 1
\end{array}\right] \\
C_{1}=\left[\begin{array}{rrr}
1 & 0 & 0 \\
0 & 1 & 0 \\
0 & 0 & \sqrt{2}
\end{array}\right], C_{2}=\left[\begin{array}{rrr}
\sqrt{2} & 0 & 0 \\
0 & 1 & 0 \\
0 & 0 & 1
\end{array}\right]
\end{gathered}
$$

Nosso objetivo inicial é determinar uma regra de comutação de tal forma que $x=0$ seja um ponto de equilíbrio globalmente assintoticamente estável. É importante notar que cada subsistema isolado é instável.

A solução ótima do problema de otimização

$$
\min _{i \in \mathbb{K}} \inf _{P_{i}>0}\left\{\operatorname{tr}\left(G^{\prime} P_{i} G\right):(31)\right\}
$$

com $\gamma=200$, foi determinada sem dificuldades tendo em vista que ele se reduz a dois problemas independentes descritos por LMIs. Desta forma determinamos as matrizes simétricas definidas positivas

$$
\begin{aligned}
& P_{1}=\left[\begin{array}{lll}
3.5511 & 3.5765 & 0.7500 \\
3.5765 & 6.8926 & 2.0458 \\
0.7500 & 2.0458 & 2.8212
\end{array}\right] \\
& P_{2}=\left[\begin{array}{lll}
3.5611 & 3.5867 & 0.7603 \\
3.5867 & 6.8927 & 2.0358 \\
0.7603 & 2.0358 & 2.7908
\end{array}\right]
\end{aligned}
$$

bem como o limitante superior 8.8925. A partir das matrizes $P_{1}$ e $P_{2}$ a regra de comutação (6) foi implementada. As trajetórias das variáveis de estado $x(t) \in \mathbb{R}^{3}$ em função do tempo, considerando uma pertubação impulsiva do tipo $w(t)=e_{1} \delta(t)$ com $e_{1}$ sendo a primeira coluna da matriz identidade, são apresentadas na Figura 1. Em seguida, o pro- blema de otimização

$$
\max _{i \in \mathbb{K}} \sup _{P_{i}>0}\left\{\operatorname{tr}\left(G^{\prime} P_{i} G\right):(38)\right\}
$$

com $\gamma=200$ também foi resolvido para determinar o limitante inferior 4.2503 . Com base nestes resultados podemos afirmar que $4.2503 \leq \Theta(\sigma) \leq 8.8925$ sendo $\sigma$ a regra de comutação (6) determinada com as matrizes $P_{1}$ e $P_{2}$. Via simulação numérica determinamos $\Theta(\sigma)=4.9730$, o que mostra uma maior precisão do limitante inferior. Por outro lado, podemos também ressaltar que muito embora o problema de controle ótimo $\inf _{\sigma} \Theta(\sigma)$, restrito ao conjunto de todas as regras de comutação estabilizantes, seja praticamente impossível de ser resolvido, sua solução $\sigma^{*}$ satisfaz $4.2503 \leq \Theta\left(\sigma^{*}\right) \leq 4.9730$. Isto coloca em evidência a qualidade do resultado obtido.

\section{SÍNTESE DE CONTROLE}

Como veremos, não há grandes dificuldades para projetar, simultaneamente, um controle via realimentação de estado e uma regra de comutação que minimizam o custo garantido introduzido anteriormente. Neste sentido, considere o sistema

$$
\begin{aligned}
\dot{x}(t) & =A_{\sigma(t)} x(t)+B_{\sigma(t)} u(t)+G_{\sigma(t)} w(t) \\
\xi(t) & =C_{\sigma(t)} x(t)+D_{\sigma(t)} u(t)
\end{aligned}
$$

com condições iniciais nulas, em que $x(t) \in \mathbb{R}^{n}$ é o estado, $u(t) \in \mathbb{R}^{r}$ é o controle, $w(t) \in \mathbb{R}^{m}$ é a entrada externa impulsiva e $\xi(t) \in \mathbb{R}^{p}$ é a saída controlada. Como anteriormente comentado, a regra de comutação $\sigma(t)=i \in \mathbb{K}$ seleciona um subsistema definido por $\left(A_{i}, B_{i}, G_{i}, C_{i}, D_{i}\right)$. Nosso objetivo é determinar uma lei de controle da forma

$$
u(t)=K_{\sigma(t)} x(t)
$$

e uma regra de comutação $\sigma(x(t))=i \in \mathbb{K}$ que seleciona, a cada instante de tempo $t \geq 0$, um elemento do conjunto $\mathcal{K}=\left\{K_{1}, \cdots, K_{N}\right\}$. Aplicando (46) em (44)-(45), o sistema em malha fechada pode ser representado pelas equações no espaço de estado

$$
\begin{aligned}
\dot{x}(t) & =\left(A_{\sigma(t)}+B_{\sigma(t)} K_{\sigma(t)}\right) x(t)+G_{\sigma(t)} w(t) \\
\xi(t) & =\left(C_{\sigma(t)}+D_{\sigma(t)} K_{\sigma(t)}\right) x(t)
\end{aligned}
$$

com condições iniciais nulas. Trata-se de um sistema com a mesma estrutura daquele já tratado. A única diferença reside na presença dos ganhos de controle a serem determinados.

Teorema 7 Se existirem dois conjuntos de matrizes simétricas definidas positivas $\left\{X_{1}, \cdots, X_{N}\right\}, Z_{i j} \forall i \neq j \in \mathbb{K} \times \mathbb{K}$, um conjunto de matrizes $\left\{L_{1}, \cdots, L_{N}\right\}$ e uma matriz $\Pi \in$ 
$\mathcal{M}$ tais que as seguintes desigualdades sejam satisfeitas

$$
\begin{gathered}
{\left[\begin{array}{cc}
\mathcal{L}_{i}+\sum_{j \neq i=1}^{N} \pi_{j i} Z_{i j} & X_{i} C_{i}^{\prime}+L_{i}^{\prime} D_{i}^{\prime} \\
\bullet & -I
\end{array}\right]<0, i \in \mathbb{K}} \\
{\left[\begin{array}{cc}
Z_{i j}+X_{i} & X_{i} \\
\bullet & X_{j}
\end{array}\right]>0, i \neq j \in \mathbb{K} \times \mathbb{K}} \\
{\left[\begin{array}{cc}
W_{i} & G_{\sigma(0)}^{\prime} \\
\bullet & X_{i}
\end{array}\right]>0, i \in \mathbb{K}}
\end{gathered}
$$

com $\mathcal{L}_{i}=A_{i} X_{i}+B_{i} L_{i}+X_{i} A_{i}^{\prime}+L_{i}^{\prime} B_{i}^{\prime}$, então a regra de comutação (6) com $P_{i}=X_{i}^{-1}$ e os ganhos de realimentação $K_{i}=L_{i} X_{i}^{-1}$ fazem com que o sistema em malha fechada seja globalmente assintoticamente estável. Ademais, a seguinte desigualdade

$$
\Theta(\sigma)<\min _{i \in \mathbb{K}} \operatorname{tr}\left(W_{i}\right)
$$

é verdadeira.

Prova: Admitindo que as desigualdades matriciais (49), (50) e (51) sejam válidas, levando em conta que $K_{i}=$ $L_{i} X_{i}^{-1}$, aplicando o Complemento de Schur em (49) e multiplicando ambos os lados por $P_{i}=X_{i}^{-1}$, obtemos

$$
\begin{aligned}
P_{i} \mathcal{L}_{i} P_{i} & +\sum_{j \neq i=1}^{N} \pi_{j i} P_{i} Z_{i j} P_{i}+ \\
& +\left(C_{i}+D_{i} K_{i}\right)^{\prime}\left(C_{i}+D_{i} K_{i}\right)<0
\end{aligned}
$$

para todo $i \in \mathbb{K}$. Em seguida, aplicando o Complemento de Schur em (50) e posteriormente multiplicando ambos os lados por $P_{i}$, obtemos

$$
P_{i} Z_{i j} P_{i}>P_{j}-P_{i}
$$

Utilizando as desigualdades (53) e (54) e lembrando que $\sum_{j \neq i=1}^{N} \pi_{j i}=-\pi_{i i}$, temos

$$
P_{i} \mathcal{L}_{i} P_{i}+\sum_{j=1}^{N} \pi_{j i} P_{j}+\left(C_{i}+D_{i} K_{i}\right)^{\prime}\left(C_{i}+D_{i} K_{i}\right)<0
$$

para todo $i \in \mathbb{K}$. Como estas desigualdades, com $P_{i} \mathcal{L}_{i} P_{i}=$ $\left(A_{i}+B_{i} K_{i}\right)^{\prime} P_{i}+P_{i}\left(A_{i}+B_{i} K_{i}\right)$, representam a aplicação do Teorema 3 no sistema em malha fechada (47)-(48) concluímos que ele é globalmente assintoticamente estável e que $\Theta(\sigma)<\min _{i \in \mathbb{K}} \operatorname{tr}\left(G_{\sigma(0)}^{\prime} P_{i} G_{\sigma(0)}\right)$ junto com (51) permitem obter (52), o que prova o teorema proposto.

Podemos utilizar um resultado alternativo, porém mais conservador, para tornar o problema apresentado no Teorema 7 possível de ser resolvido através de LMIs e de uma busca unidimensional, tal como foi feito anteriormente. O corolário a seguir apresenta as LMIs resultantes.
Corolário 8 Se existirem um conjunto de matrizes simétricas definidas positivas $\left\{X_{1}, \cdots, X_{N}\right\}$, um conjunto de matrizes $\left\{L_{1}, \cdots, L_{N}\right\}$ e um escalar positivo $\gamma$ satisfazendo as seguintes desigualdades modificadas de Lyapunov-Metzler

$$
\left[\begin{array}{ccc}
\mathcal{L}_{i}-\gamma X_{i} & X_{i} C_{i}^{\prime}+L_{i}^{\prime} D_{i}^{\prime} & \gamma X_{i} \\
\bullet & -I & 0 \\
\bullet & \bullet & -\gamma X_{j}
\end{array}\right]<0,
$$

para todo $i \neq j \in \mathbb{K} \times \mathbb{K}$

$$
\left[\begin{array}{cc}
W_{i} & G_{\sigma(0)}^{\prime} \\
\bullet & X_{i}
\end{array}\right]>0, i \in \mathbb{K}
$$

com $\mathcal{L}_{i}=A_{i} X_{i}+B_{i} L_{i}+X_{i} A_{i}^{\prime}+L_{i}^{\prime} B_{i}^{\prime}$, então a regra de comutação (6) com $P_{i}=X_{i}^{-1}$ e os ganhos de realimentação $K_{i}=L_{i} X_{i}^{-1}$ fazem com que o sistema em malha fechada seja globalmente assintoticamente estável e a desigualdade (52) seja verdadeira.

Prova: Imediata e será, portanto, omitida.

Para o cálculo do limitante inferior basta verificar que o Teorema 5 ou o Corolário 6 podem ser aplicados imediatamente para o sistema em malha fechada. Isto é, as matrizes $A_{q}$ e $C_{q}$ devem ser substituídas pelas matrizes $A_{q}+B_{q} K_{q}$ e $C_{q}+D_{q} K_{q}$, respectivamente. Observe que os referidos resultados permitem determinar limitantes inferiores para o custo $\Theta(\sigma)$ correspondentes aos ganhos de realimentação $K_{q}$ já determinados. Como acabamos de verificar através do Teorema 7 e do Corolário 8, a dificuldade de síntese está concentrada no cálculo de $\Theta(\sigma)$ e não na determinação dos ganhos de realimentação que definem o controle $u(t)=K_{\sigma(t)} x(t)$. $\mathrm{O}$ exemplo dado a seguir ilustra os resultados apresentados nesta seção.

Exemplo 2 : Foi inspirado em um exemplo tratado em (Xie e Wang, 2004). O objetivo é aplicar o procedimento de síntese de controle do Corolário 8 ao sistema (44)-(45) com as respectivas matrizes do seu modelo de estado dadas por

$$
\begin{gathered}
A_{1}=\left[\begin{array}{rr}
-5 & 0 \\
0 & 8
\end{array}\right], A_{2}=\left[\begin{array}{rr}
3 & 0 \\
0 & -2
\end{array}\right] \\
G_{1}=\left[\begin{array}{l}
2 \\
2
\end{array}\right], G_{2}=\left[\begin{array}{l}
1 \\
1
\end{array}\right], B_{1}=\left[\begin{array}{l}
4 \\
0
\end{array}\right], B_{2}=\left[\begin{array}{l}
0 \\
2
\end{array}\right], \\
C_{1}=\left[\begin{array}{ll}
2 & 0 \\
0 & 0
\end{array}\right], C_{2}=\left[\begin{array}{ll}
0 & 4 \\
0 & 0
\end{array}\right], D_{1}=D_{2}=\left[\begin{array}{l}
0 \\
2
\end{array}\right]
\end{gathered}
$$

Cabe notar que cada subsistema isolado é instável e não controlável. Mesmo assim, será possível determinar uma regra de comutação estabilizante. Fixando $\gamma=500$, o problema de otimização

$$
\min _{i \in \mathbb{K}} \inf _{X_{i}, L_{i}, W_{i}}\left\{\operatorname{tr}\left(W_{i}\right):(56)-(57)\right\}
$$




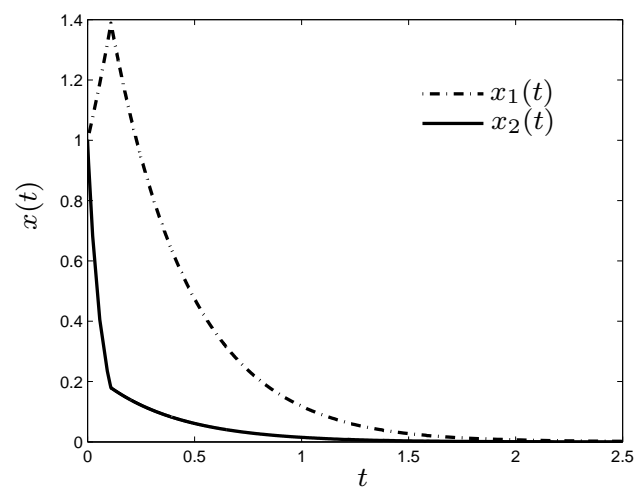

Figura 2: Estados do sistema - Exemplo 2

foi resolvido considerando $\sigma(0)=1$ e $\sigma(0)=2$. A solução com menor custo 14.3278 , que define um limitante superior para $\Theta(\sigma)$, correspondente a $\sigma(0)=2$ é dada por

$$
\begin{aligned}
P_{1} & =\left[\begin{array}{rr}
0.6232 & -0.0001 \\
-0.0001 & 14.1500
\end{array}\right] \\
P_{2} & =\left[\begin{array}{rr}
0.6308 & -0.0001 \\
-0.0001 & 13.6972
\end{array}\right] \\
K_{1} & =\left[\begin{array}{ll}
-0.6232 & 0.0001
\end{array}\right] \\
K_{2} & =\left[\begin{array}{ll}
0.0001 & -6.8486
\end{array}\right]
\end{aligned}
$$

A Figura 2 apresenta as trajetórias dos estados do sistema em malha fechada $x(t) \in \mathbb{R}^{2}$ que convergem para zero em um intervalo de tempo menor do que 2 segundos. Para os mesmos ganhos de realimentação, com o Corolário 6, determinamos um limitante inferior de $\Theta(\sigma)$ igual a 7.2892. Portanto, este exemplo ilustra que mesmo com todos os subsistemas instáveis e não controláveis, as condições do Corolário 8 permitem determinar uma lei de controle estabilizante, cujo custo pertence à faixa $7.2892 \leq \Theta(\sigma) \leq 14.3278$. Na verdade, via simulação numérica o valor exato $\Theta(\sigma)=7.8812$ foi determinado, o que mais uma vez comprova a qualidade do resultado, sobretudo a precisão do limitante inferior. Por outro lado, aplicando as condições do Corolário 8 com os ganhos de realimentação nulos, deixando atuar apenas a regra de comutação $\sigma(t)$, não é possível fazer com que a origem seja globalmente assintoticamente estável. Portanto, este exemplo mostra a importância da síntese conjunta de ambas as variáveis de controle, a saber, a regra de comutação e os ganhos de realimentação de estado $\left\{K_{1}, \cdots, K_{N}\right\}$.

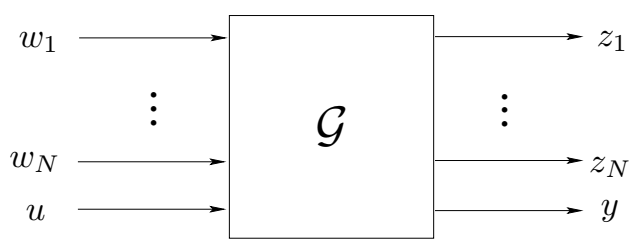

Figura 3: Estrutura do sistema multiobjetivo

\section{CONTROLE $\mathcal{H}_{2}$ MULTIOBJETIVO}

Nesta seção, aplicamos os resultados desenvolvidos anteriormente em problemas de controle $\mathcal{H}_{2}$ multiobjetivo tendo em vista a real possibilidade de tratarmos sistemas com diversos critérios de desempenho possivelmente conflitantes. Considere o sistema linear invariante no tempo $\mathcal{G}$ com múltiplas entradas e múltiplas saídas apresentado na Figura 3 e descrito pelas seguintes equações de estado

$$
\begin{aligned}
\dot{x} & =A x+H w+B u \\
z & =C x+D u \\
y & =E x+G w
\end{aligned}
$$

com condições iniciais nulas. Os vetores $x, u, w, z$ e $y$ representam o estado, o controle, a entrada externa, a saída controlada e a saída medida, respectivamente. As matrizes $H, C$ e $D$ são particionadas de acordo com a partição do vetor de entrada $w^{\prime}=\left[w_{1}^{\prime} \cdots w_{N}^{\prime}\right]$ e do vetor de saída $z^{\prime}=\left[\begin{array}{lll}z_{1}^{\prime} & \cdots & z_{N}^{\prime}\end{array}\right]$ da seguinte forma

$$
\begin{aligned}
H & =\left[\begin{array}{lll}
H_{1} & \cdots & H_{N}
\end{array}\right] \\
C^{\prime} & =\left[\begin{array}{lll}
C_{1}^{\prime} & \cdots & C_{N}^{\prime}
\end{array}\right] \\
D^{\prime} & =\left[\begin{array}{lll}
D_{1}^{\prime} & \cdots & D_{N}^{\prime}
\end{array}\right]
\end{aligned}
$$

A saída medida $y$ é conectada à entrada de controle $u$ através de uma função de transferência $K(s)$ definida pelo controlador de tal forma que para cada $i \in \mathbb{K}$ denominamos $T_{i}(s)$ a função de transferência em malha fechada entre a entrada $w_{i}$ e a saída $z_{i}$. O problema $\mathcal{H}_{2}$ multiobjetivo consiste na determinação de uma única função de transferência $K(s)$ de forma a estabilizar o sistema em malha fechada e minimizar o vetor $J(K)$

$$
J(K)=\left[\begin{array}{lll}
\left\|T_{1}\right\|_{2}^{2} & \cdots & \left\|T_{N}\right\|_{2}^{2}
\end{array}\right]
$$

Esta minimização não é fácil, uma vez que geralmente, a minimização dos critérios $\left\|T_{i}\right\|_{2}^{2}$ para $i \in \mathbb{K}$ apresenta objetivos conflitantes, ou seja, a função de transferência $K(s)$ que minimiza cada um deles não é a mesma. A solução deste problema foi estudada por vários autores, veja (Khargonekar e Rotea, 1991) e suas referências. A estratégia utilizada por (Khargonekar e Rotea, 1991) foi a utilização da parametrização de Youla, que permite expressar as quantidades $\left\|T_{i}\right\|_{2}^{2}$ 
como funções afins de uma variável $Q \in R H_{\infty}$. Graças à convexidade de $\left\|T_{i}(Q)\right\|_{2}^{2}$ com relação a $Q \in R H_{\infty}$, o conjunto de todas as soluções ótimas de Pareto é obtido a partir do seguinte problema de otimização escalar

$$
\inf _{Q \in R H_{\infty}} \sum_{i=1}^{N} \lambda_{i}\left\|T_{i}(Q)\right\|_{2}^{2}
$$

com $\lambda \in \mathbb{R}^{N}$ representando um elemento do conjunto convexo $\Lambda$ definido em (3). Para cada valor de $\lambda \in \Lambda$ dado, o problema (66) fornece uma das soluções ótimas de Pareto, sendo possível obter o valor ótimo de $Q^{o} \in R H_{\infty}$ e, por consequiência, o controlador ótimo $K^{o}$, tal que a conexão $u=K^{o} * y$ estabiliza o sistema em malha fechada e minimiza a função objetivo escalar $\sum_{i=1}^{N} \lambda_{i}^{o}\left\|z_{i}\right\|_{2}^{2}$, com $z_{i}$ sendo a resposta ao impulso do $i$-ésimo canal com entrada $w_{i}$ e saída $z_{i}$ para todo $i \in \mathbb{K}$. O vetor $\lambda^{o}$ é definido a priori de forma a selecionar uma solução de Pareto escolhida.

Nesta seção, pretendemos empregar os resultados anteriores para resolver o problema multiobjetivo exposto em (65), a partir de uma formulação mais abrangente que passamos a discutir. Supondo que o sistema (59)-(61) possua apenas uma entrada e múltiplas saídas, propomos uma lei de controle da forma $u(t)=K_{\sigma(t)} * y(t)$, em que $\sigma(t)$ é a regra de comutação que seleciona a cada instante de tempo um ganho de realimentação específico $K_{\sigma(t)} \in \mathcal{K}=\left\{K_{1}, \cdots, K_{N}\right\}$. Diferente do problema clássico multiobjetivo (Khargonekar e Rotea, 1991), em que o vetor $\lambda \in \Lambda$ é considerado invariante no tempo, no presente caso, $\lambda$ é variante no tempo $\lambda(t) \in \mathcal{V}$, sendo que $\mathcal{V}$ representa o conjunto de todos os vértices de $\Lambda$, e corresponde a uma variável adicional a ser determinada de forma a minimizar a função objetivo $\sum_{i=1}^{N}\left\|\sqrt{\lambda_{i}} z_{i}\right\|_{2}^{2}$. Para cada $t \geq 0$, associada ao vetor $\lambda(t) \in \mathcal{V}$, definimos a regra de comutação $\sigma(t)=i$ se $\lambda_{i}(t)=1$ e $\lambda_{j}(t)=0$ para todo $i \neq j$ e a nova saída controlada $\xi(t)=C_{\sigma(t)} x(t)+D_{\sigma(t)} u(t)$. O novo problema de otimização a ser resolvido é

$$
\inf _{(\sigma, u) \in \mathcal{Y}}\|\xi\|_{2}^{2}
$$

sendo que $\mathcal{Y}$ representa o conjunto das informações disponíveis para a realimentação, ou seja $\mathcal{Y}=\{y(\tau): \forall \tau \leq t\}$. O problema (67) não é simples de ser resolvido devido à natureza variante no tempo da lei de comutação $\sigma(t)$. Assim, em (Eker e Malmborg, 1999) foi proposta uma estratégia para resolver (67) composta de duas etapas. A primeira consiste em encontrar o valor de $K_{i}$ para cada $\sigma(t)=i$ fixo

$$
K_{i}=\arg \inf _{(\sigma, u) \in \mathcal{Y}}\left\{\|\xi\|_{2}^{2}: \sigma=i\right\}, \forall i \in \mathbb{K}
$$

A segunda consiste na determinação da regra de comutação

$$
\sigma(t)=\arg \min _{i \in \mathbb{K}} \bar{v}_{i}(x(t))
$$

a partir da função custo $\bar{v}_{i}(x)$ associada ao problema de otimização resolvido na primeira etapa.

No contexto deste trabalho, propomos substituir o problema de otimização (67), pela minimização de um limitante superior adequado para $\|\xi\|_{2}^{2}$ que fornece a regra de comutação e um conjunto de ganhos de realimentação $\mathcal{K}=$ $\left\{K_{1}, \cdots, K_{N}\right\}$. Neste sentido, considerando o sistema (59)(61) com a nova saída $\xi$, admitindo que todos os estados estejam disponíveis para realimentação, o que implica em $E=I, G=0$ e substituindo $u(t)=K_{\sigma(t)} x(t)$ em (59)(61), obtemos

$$
\begin{aligned}
\dot{x} & =\left(A+B K_{\sigma}\right) x+H w \\
\xi & =\left(C_{\sigma}+D_{\sigma} K_{\sigma}\right) x
\end{aligned}
$$

com condições iniciais nulas. Note que este sistema em malha fechada tem uma estrutura idêntica àquele dado em (47)(48). Portanto, a solução do problema exposto pode ser obtida aplicando-se o resultado do Corolário 8 que fornece

$$
J^{o}=\min _{i \in \mathbb{K}} \inf _{\gamma>0} \inf _{\left(X_{i}, W_{i}, L_{i}\right) \in \Phi(\gamma)} \operatorname{Tr}\left(W_{i}\right)
$$

sendo $\Phi(\gamma)$ o conjunto de todas as matrizes $\left(X_{i}, W_{i}, L_{i}\right)$, $i \in \mathbb{K}$ satisfazendo as desigualdades (56) e (57) para $\gamma>0$ fixo, de tal maneira que $\inf _{(\sigma, u) \in \mathcal{Y}}\|\xi\|_{2}^{2} \leq J^{o}$ pois, para a mesma regra de comutação e os mesmos ganhos de realimentação, sabemos que $\|\xi\|_{2}^{2}=\Theta(\sigma)$. Devemos notar que o problema (72) é equivalente a $N$ problemas independentes que podem ser resolvidos fazendo uma busca unidimensional em $\gamma$ e resolvendo um conjunto de LMIs.

Teorema 9 Para o caso de realimentação de estado, as seguintes afirmações são verdadeiras:

a) O custo $J^{o}$ é menor ou igual ao custo da solução proposta em (Eker e Malmborg, 1999).

b) O custo $J^{o}$ é menor ou igual ao custo de qualquer solução de Pareto dada em (Khargonekar e Rotea, 1991).

Prova: Considerando $\gamma>0$ arbitrariamente pequeno, as restrições (56) são desacopladas e portanto

$$
J^{o} \leq \min _{i \in \mathbb{K}} \inf _{P_{i}>0, K_{i}} \operatorname{Tr}\left(H^{\prime} P_{i} H\right)
$$

em que $P_{i}>0$ e $K_{i}$ são tais que

$$
A_{k i}^{\prime} P_{i}+P_{i} A_{k i}+C_{k i}^{\prime} C_{k i}<0
$$

com $A_{k i}=A+B K_{i}$ e $C_{k i}=C+D K_{i}$, para todo $i \in \mathbb{K}$. Logo, da desigualdade (73) obtemos

$$
J^{o} \leq \min _{i \in \mathbb{K}} \inf _{K_{i}}\left\|C_{k i}\left(s I-A_{k i}\right)^{-1} H\right\|_{2}^{2}
$$


que prova o item $a$ ), uma vez que a solução de (75) em relação a $K_{i}$ satisfaz (68) e $\bar{v}_{i}(x)=x^{\prime} P_{i} x$. Para provar o item b) considere $K$ um ganho estabilizante qualquer e introduza no problema (72) as restrições adicionais $K X_{i}=L_{i}$, para todo $i \in \mathbb{K}$. Com $\gamma$ positivo arbitrariamente pequeno, (73) e (74) são válidas para $K=K_{1}=\cdots=K_{N}$ e, conseqüentemente, de (75), temos

$$
J^{o} \leq \inf _{K} \sum_{i=1}^{N} \lambda_{i}\left\|C_{k i}\left(s I-A_{k i}\right)^{-1} H\right\|_{2}^{2}
$$

para todo $\lambda \in \Lambda$ o que prova o teorema proposto.

A prova do Teorema 9 deixa claro que os métodos propostos em (Khargonekar e Rotea, 1991) e (Eker e Malmborg, 1999) são comparáveis ao deste artigo considerando o parâmetro $\gamma$ arbitrariamente pequeno. Logo, é de se esperar que com um grau de liberdade adicional, introduzido por $\gamma>0$, seja possível determinar uma solução com menor custo. Esta conclusão é natural pois em relação ao método (Eker e Malmborg, 1999) temos um grau de liberdade adicional (parâmetro $\gamma>0$ ) e, em relação a (Khargonekar e Rotea, 1991) temos vários graus de liberdade adicionais $(\gamma>0$ e $N$ diferentes ganhos $\left.K_{1}, \cdots, K_{N}\right)$. O exemplo a seguir ilustra os aspectos teóricos discutidos nesta seção.

Exemplo 3 : Este exemplo foi extraído de (Geromel e Deaecto, 2007). Considere o sistema (59)-(61), com $H=I$ e as matrizes

$$
\begin{gathered}
A=\left[\begin{array}{lll}
0 & 1 & 0 \\
0 & 0 & 1 \\
0 & 0 & 0
\end{array}\right], B=\left[\begin{array}{l}
0 \\
0 \\
1
\end{array}\right] \\
C_{1}=\left[\begin{array}{lll}
4 & 0 & 0 \\
0 & 4 & 0 \\
0 & 0 & 0
\end{array}\right], D_{1}=\left[\begin{array}{l}
0 \\
0 \\
1
\end{array}\right] \\
C_{2}=\left[\begin{array}{lll}
1 & 0 & 0 \\
0 & 1 & 0 \\
0 & 0 & 0
\end{array}\right], D_{2}=\left[\begin{array}{l}
0 \\
0 \\
8
\end{array}\right]
\end{gathered}
$$

que representa um triplo integrador com dois critérios conflitantes. Desejamos resolver o problema $\mathcal{H}_{2}$ multiobjetivo utilizando cada um dos métodos discutidos e comparando-os entre si.

- Método I (Khargonekar e Rotea, 1991) - A Figura 4 apresenta o conjunto de todas as soluções de Pareto do problema multiobjetivo. Foi verificado que elas têm custos entre 51.33 para $\lambda=\left[\begin{array}{ll}1 & 0\end{array}\right]^{\prime}$ e 139.49 para $\lambda=\left[\begin{array}{lll}0.4 & 0.6\end{array}\right]^{\prime}$.

- Método II (Eker e Malmborg, 1999) - A solução ótima do primeiro critério tem um custo igual a 51.33 enquanto que o valor mínimo do segundo critério é 95.40. Assim sendo, o controle obtido por este método apresenta um custo não superior a 51.33 .

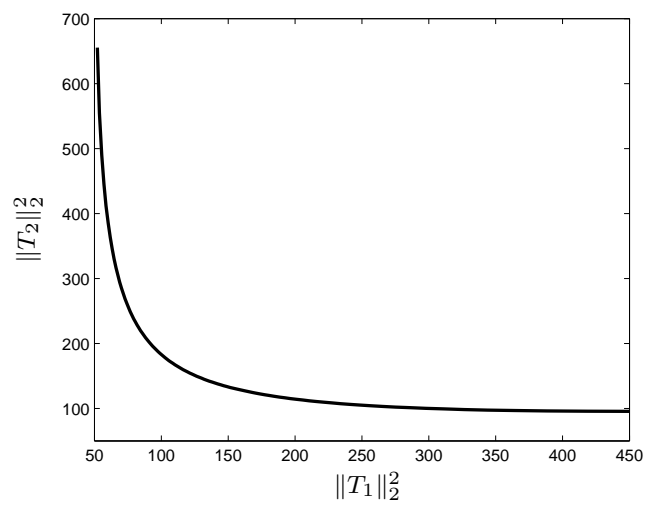

Figura 4: Soluções de Pareto

- Método Proposto - O problema (72) foi resolvido para $\gamma=\left\{10^{-5}, 10^{-1}, 1,2,10\right\}$ obtendo-se os custos associa$\operatorname{dos} J^{o}=\{51.33,56.39,57.70,51.89,42.16\}$. Note que para $\gamma$ muito pequeno o custo obtido é praticamente igual ao do método II. Para $\gamma$ superior a 2.2 os custos obtidos são sempre menores que os dos métodos I e II, indicando que o grau de liberdade adicional devido ao parâmetro $\gamma$ resulta, de fato, em um sistema em malha fechada com melhor desempenho. Se o problema (72) fosse resolvido para os mesmos valores de $\gamma$, mas considerando o ganho $K_{i}$ conhecido e igual ao ganho ótimo de cada critério isolado para $i=1 \mathrm{e} 2$, os custos obtidos seriam $J^{o}=\{51.33,58.87,92.26,93.37,89.91\}$. Note que eles seriam maiores que os obtidos pelos métodos anteriores, indicando a importância de se projetar as duas variáveis de controle $K_{\sigma(t)}$ e $\sigma(t)$ conjuntamente.

A Figura 5 apresenta as trajetórias da variável de estado $x_{3}(t)$ a partir da condição inicial $x(0)=\left[\begin{array}{lll}1 & 1 & 1\end{array}\right]^{\prime}$, com o controle ótimo relativo a cada critério isolado, o controle do método II e com aquele obtido pelo método aqui proposto para $\gamma=$ 10. Os custos correspondentes são 102.16, 194.31, $102.16 \mathrm{e}$ 57.25 , respectivamente.

\section{CONCLUSÃO}

Neste trabalho, analisamos diversos aspectos de sistemas lineares com comutação. Dentre eles devemos citar o estudo de estabilidade assintótica global e a determinação de um custo garantido que se aplica quando o sistema estiver sujeito à perturbações externas impulsivas. Este último resultado reduz-se à norma $\mathcal{H}_{2}$ quando não ocorrem comutações. As diversas condições são expressas por um conjunto de desigualdades de Lyapunov-Metzler que possuem natureza não-convexa sendo, portanto, de difícil solução. Para 


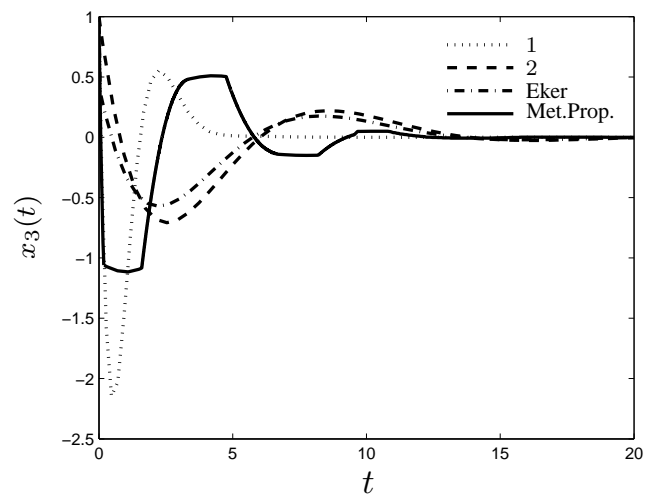

Figura 5: Simulação de $x_{3}(t)$

contornar esta dificuldade propomos um método alternativo que substitui a solução de desigualdades não-convexas por uma busca unidimensional e a solução de um conjunto de LMIs. Para evidenciar a qualidade do resultado final, calculamos limitantes inferiores para o critério de desempenho adotado. Um ponto interessante e ilustrado através de um exemplo é a possibilidade de determinar um controle estabilizante mesmo que cada subsistema isolado seja instável e não controlável. A teoria desenvolvida foi utilizada com sucesso para propor uma solução alternativa para problemas $\mathcal{H}_{2}$ multiobjetivo. Neste particular contexto, o método proposto fornece resultados melhores que os existentes na literatura para tratar esta classe de problemas. Um tópico interessante para pesquisas futuras consiste na obtenção de resultados similares para sistemas com comutação e realimentação de saída. Neste caso, o objetivo principal continua sendo a determinação de um conjunto de controladores dinâmicos e uma lei de comutação que sejam capazes de assegurar a estabilidade assintótica global do sistema em malha fechada. A dificuldade central é que, não sendo o estado disponível, as regras de comutação aqui apresentadas não podem ser adotadas. Uma primeira abordagem deste problema foi feita em (Geromel et al., 2008).

\section{APÊNDICE}

Seja $f(x, i): \mathbb{R}^{n} \times \mathbb{K} \rightarrow \mathbb{R}$. Por hipótese, $f(x, i)$ tem derivadas parciais $\partial f / \partial x_{i}$ contínuas para todo $i \in \mathbb{K}$. Defina a função $\nu(x): \mathbb{R}^{n} \rightarrow \mathbb{R}$ como sendo

$$
\nu(x)=\min _{i \in \mathbb{K}} f(x, i)=\min _{\lambda \in \Lambda} \sum_{i=1}^{N} \lambda_{i} f(x, i)
$$

Trata-se de uma função contínua mas que não é necessariamente diferenciável nos pontos em que o conjunto

$$
Z(x)=\{i \in \mathbb{K}: \nu(x)=f(x, i)\} \subset \mathbb{K}
$$

é composto por mais de um elemento. A derivada direcional à direita da função (77) em um ponto $x \in \mathbb{R}^{n} \mathrm{e}$ em uma direção $d \in \mathbb{R}^{n}$ é definida na forma

$$
D^{+} \nu(x, d)=\lim _{h \rightarrow 0^{+}} \frac{\nu(x+h d)-\nu(x)}{h}
$$

e é calculada com o auxílio do Teorema de Danskin cuja prova pode ser encontrada em (Lasdon, 1970), pag. 420.

Teorema de Danskin A derivada direcional da função $\nu(x)$ existe em qualquer ponto $x \in \mathbb{R}^{n}$, em qualquer direção $d \in$ $\mathbb{R}^{n}$ e é dada por

$$
D^{+} \nu(x, d)=\min _{l \in Z(x)} \nabla f(x, l)^{\prime} d
$$

A prova deste importante resultado mostra que a derivada direcional $D^{+} \nu(x, d)$ dada acima é conseguida tendo em vista que os limites lim sup e lim inf do lado direito de (79) coincidem.

Suponha agora que $x(t), t \geq 0$ seja uma trajetória qualquer do sistema linear $\dot{x}(t)=A x(t)$. Para $h \rightarrow 0^{+}$, temos $x(t+$ $h)=x(t)+h \dot{x}(t)=x(t)+h A x(t)$ e portanto

$$
\begin{aligned}
D^{+} \nu(x(t)) & =\lim _{h \rightarrow 0^{+}} \frac{\nu(x(t+h))-\nu(x(t))}{h} \\
& =D^{+} \nu(x(t), A x(t)) \\
& =\min _{l \in Z(x(t))} \nabla f(x(t), l)^{\prime} A x(t)
\end{aligned}
$$

No caso particular tratado neste artigo que corresponde a escolher $f(x, i)=x^{\prime} P_{i} x$ com $P_{i}$ matrizes simétricas definidas positivas para todo $i \in \mathbb{K}$, obtemos

$$
D^{+} \nu(x(t))=\min _{l \in Z(x(t))} x(t)^{\prime}\left(A^{\prime} P_{l}+P_{l} A\right) x(t)
$$

Este procedimento pode também ser aplicado para calcular a derivada direcional da função

$$
\vartheta(x)=\max _{i \in \mathbb{K}} f(x, i)=\max _{\lambda \in \Lambda} \sum_{i=1}^{N} \lambda_{i} f(x, i)
$$

De fato, definindo $\mathcal{Z}(x)=\{i \in \mathbb{K}: \vartheta(x)=f(x, i)\} \subset \mathbb{K}$ e reescrevendo-a de forma equivalente

$$
\vartheta(x)=-\min _{i \in \mathbb{K}}-f(x, i)
$$

o Teorema de Danskin fornece

$$
\begin{aligned}
D^{+} \vartheta(x, d) & =-\min _{l \in \mathcal{Z}(x)}-\nabla f(x, l)^{\prime} d \\
& =\max _{l \in \mathcal{Z}(x)} \nabla f(x, l)^{\prime} d
\end{aligned}
$$


permitindo concluir que, em particular, para as funções quadráticas $f(x, i)=x^{\prime} P_{i} x$ com $P_{i}$ matrizes simétricas definidas positivas para todo $i \in \mathbb{K}$, temos

$$
D^{+} \vartheta(x(t))=\max _{l \in \mathcal{Z}(x(t))} x(t)^{\prime}\left(A^{\prime} P_{l}+P_{l} A\right) x(t)
$$

Estes resultados são aplicados em diversos pontos deste artigo, sobretudo na prova do Teorema 1 e na discussão colocada em seguida.

\section{AGRADECIMENTOS}

Agradecemos à Fapesp (Fundação de Amparo à Pesquisa do Estado de São Paulo) e ao CNPq (Conselho Nacional de Desenvolvimento Científico e Tecnológico) pelo apoio financeiro e aos revisores, cujas sugestões certamente contribuíram para o aprimoramento deste artigo.

\section{REFERÊNCIAS}

Boyd, S. P., Ghaoui, L. E., Feron, E. e Balakrishnan, V. (1994). Linear matrix inequalities in system and control theory, SIAM, Philadelphia.

Colaneri, P., Geromel, J. C. e Locatelli, A. (1997). Control theory and design: an $\mathrm{RH}_{2}$ and $\mathrm{RH}_{\infty}$ viewpoint, Academic Press Inc., London.

Daafouz, J. e Bernussou, J. (2001). Parameter dependent Lyapunov functions for discrete time systems with time varying parametric uncertainties, Systems \& Contr. Letters 43: 355-359.

Deaecto, G. S. (2007). Síntese de controle para sistemas dinâmicos com comutação, tese de mestrado, Universidade Estadual de Campinas - FEEC, Campinas, São Paulo.

DeCarlo, R. A., Branicky, M. S., Pettersson, S. e Lennartson, B. (2000). Perspectives and results on the stability and stabilization of hybrid systems, Proceedings of the IEEE 88: 1069-1082.

Eker, J. e Malmborg, J. (1999). Design and implementation of a hybrid control strategy, IEEE Contr. and Systems Magazine 19: 12-21.

Garg, K. M. (1998). Theory of differentiation: A unified theory of differentiation via new derivate theorems and new derivatives, Wiley-Interscience.

Geromel, J. C. e Colaneri, P. (2006a). Stability and stabilization of continuous-time switched linear systems, SIAM Journal on Contr. and Optimization 45: 470-482.
Geromel, J. C. e Colaneri, P. (2006b). Stability and stabilization of discrete-time switched systems, Int. Journal of Contr. 79: 719-728.

Geromel, J. C., Colaneri, P. e Bolzern, P. (2008). Dynamic output feedback control of switched linear systems, IEEE Trans. on Automat. Contr. 53: 720-733.

Geromel, J. C. e Deaecto, G. S. (2007). Multi-objective $\mathcal{H}_{2}$ control via switched linear systems, Proceedings of the 3rd IFAC Symposium on System, Structure and Control, SSSC-07, session WE2: Switched and jump systems, paper 3, Iguassu Falls.

Hespanha, J. P. (2005). Uniform stability of switched linear systems: extensions of LaSalle's Principle, IEEE Trans. on Automat. Contr. 43: 555-559.

Hespanha, J. P. e Morse, A. S. (2002). Switching between stabilizing controllers, Automatica 38: 1905-1917.

Ishii, H., Basar, T. e Tempo, R. (2004). Randomized algorithms for synthesis of switching rules of multimodal systems, IEEE Trans. on Automat. Contr. 49: 470-482.

Johansson, M. e Rantzer, A. (1998). Computation of piecewiese quadratic Lyapunov functions for hybrid systems, IEEE Trans. on Automat. Contr. 43: 555-559.

Khargonekar, P. P. e Rotea, M. A. (1991). Multiobjective optimal control of linear systems: The quadratic norm case, IEEE Trans. on Automat. Contr. 36: 14-24.

Lasdon, L. S. (1970). Optimization theory for large systems, Macmillan, NY.

Liberzon, D. (2003). Switching in systems and control, Birkhäuser.

Liberzon, D. e Morse, A. S. (1999). Basic problems in stability and design of switched systems, IEEE Contr. Systems Magazine 19: 59-70.

Shorten, R., Wirth, F., Mason, O., Wulff, K. e King, C. (2007). Stability criteria for switched and hybrid systems, SIAM Review 49: 545-592.

Spinelli, W., Bolzern, P. e Colaneri, P. (2006). Computation of lower bounds for the optimal quadratic cost of linear switched systems, Proceedings of the 2006 American Control Conference pp. 5444-5449.

Wirth, F. (2005). A converse Lyapunov theorem for linear parameter varying and linear switching systems, SIAM Journal Contr. and Optimization 44: 210-239.

Xie, G. e Wang, L. (2004). Periodical stabilization of switched linear systems, Journal of Computational and Applied Mathematics 181: 176-187. 